\title{
Rydberg Atoms on Fire
}

\section{A new experiment reveals unexpected connections between a nonequilibrium phase transition in Rydberg gases and the way fires spread through a burning forest.}

\section{by Marco Schiró ${ }^{1}$}

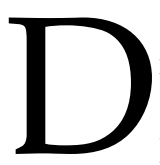
riven and dissipative systems far from thermodynamic equilibrium show a rich variety of patterns, such as the distribution of earthquakes and the patchwork of tree clusters in a forest. Sustained by a permanent input of energy, these phenomena do not follow the same energy minimization principle that organizes systems in equilibrium. Instead, driven and dissipative systems appear to spontaneously develop order through self-organized criticality - a balancing act between energy loss and energy gain. A complete understanding of how this self-organization works is lacking, partly because the relevant systems are hard to control. A new experiment by Dong-Sheng Ding and colleagues of the University of Science and Technology of China in Hefei and their collaborators at the University of Durham, UK, shows that Rydberg atoms can provide a platform for studying the mechanisms behind self-organization and nonequilibrium phase transi-

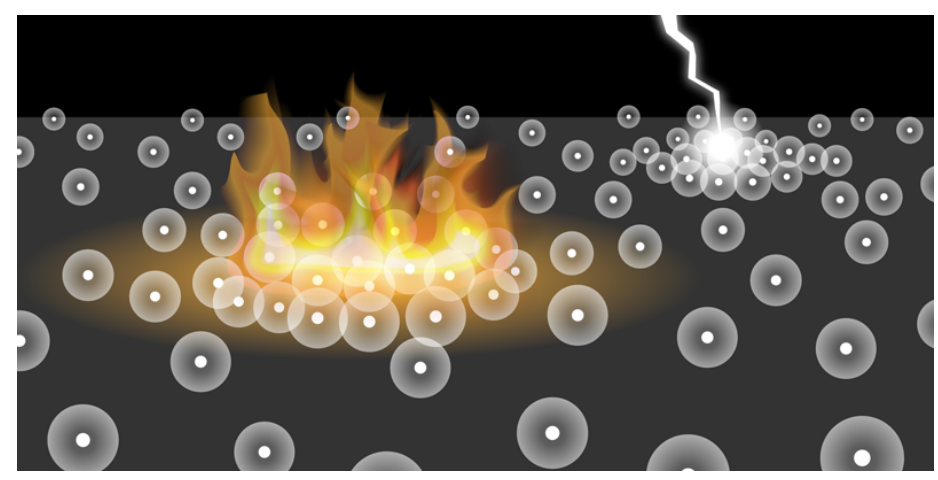

Figure 1: A system of excited atoms—called Rydberg atoms-undergoes transitions between low- and high-density phases. A new study shows that these phase transitions can be explained with a self-organized criticality model for forest fires, in which lightning strikes start fires that burn down close-packed trees. (APS/Alan Stonebraker)

${ }^{1}$ French National Center for Scientific Research (CNRS) and Institute of Physics, Collège de France, PSL Research University, Paris, France tions [1]. Remarkably, the team shows that their atomic system can be described by a self-organized criticality model for forest fires.

Physics is a matter of scales. As Nobel prize winner Kenneth Wilson put it, there is a great diversity of size and length scales in nature that are usually decoupled, such that what happens at a given scale has little or no effect on phenomena at a well-separated scale [2]. Think about water molecules in your cup of tea: their behavior is pretty much the same as it would be if they were in the ocean, since molecular interactions occur over nanometers. Molecules at larger distances have a small effect.

When this is not the case, namely when there is no characteristic length scale controlling a given physical phenomenon, things usually get interesting. No characteristic length means all length scales contribute, and the system looks the same at any scale of observation. This scale invariance means different parts of the system strongly fluctuate together in a correlated way, leading to a unique statistical signature: power-law distributions. A power-law distribution can be contrasted with a Gaussian distribution, which has a well-defined average and an exponentially small probability for rare events. Systems that obey power-law distributions, like earthquakes and neurons, experience rare events relatively often.

Scale-invariant power-law distributions are extremely common in nature, spanning a broad range of systems that are often far away from thermal equilibrium. This ubiquity suggests that a general mechanism is at play, but is there any feature that can unite all the different power-law systems? An elegant answer to this question was proposed over 30 years ago, under the name of self-organized criticality [3]. The idea is that a system with dissipation and drive will evolve toward a dynamic equilibrium that balances the energy being lost with the energy being gained. An example is a system being driven by a slow push. Rather than dissipating this energy slowly, the system releases its pent-up stress in rapid, irregular rearrangements (avalanches), whose sizes are power-law distributed. Researchers originally proposed this self-organized criticality model to explain noise spectra that scale inversely with frequency $f$. This $1 / f$ noise is found in many systems, such as heart beats and stock market data. Related examples include sandpiles [3], earthquakes, and fire spreading in a forest [4].

Self-organized criticality has evolved into a paradigm of 
complexity in science, with applications branching out well beyond physics [5]. Despite its success, much about the theory is still actively debated [6], partly because the best physical examples-such as forest fires and sand piles-resist simple characterizations. As a result, having an experimental setup to explore the physics of self-organization and criticality in a controlled way would be invaluable.

Recent work in the field of quantum simulation suggests that atomic systems might offer a platform for studying criticality. The experimental setup is one with Rydberg atoms, which are neutral atoms that are laser excited to a state with very high principal quantum number. Rydberg atoms enjoy a wealth of remarkable properties, including large dipoledipole interactions and long lifetimes, which make them perfect platforms for quantum information processing [7] and for simulation of complex quantum phases and phase transitions, often out of equilibrium [8]. Earlier this year, researchers made the first observation of self-organized criticality in a gas of Rydberg potassium atoms cooled down to microkelvin temperatures [9].

The new experiment by Ding et al. develops on this potential to use Rydberg atoms for probing criticality [1]. The team begins by trapping a thermal ensemble of rubidium atoms and laser exciting them to a Rydberg state. Scanning the wavelength of the laser they observed a sharp change in the light transmitted through the ensemble, a signature of a transition between two phases with high and low densities of Rydberg atoms. By tuning the laser wavelength precisely, they were able to explore the critical state-where the system transitions back and forth between the high- and low-density phases-in new detail. Their measurements revealed that these phase transitions are highly sensitive to fluctuations in the atomic interactions. There were other indications that the atoms were exhibiting self-organized criticality, including the transmission signal varying in time with a characteristic $1 / f$ power-law spectrum (where $f$ is the inverse-time frequency) and the transition from low to high density occurring through an avalanche process whose sizes were power-law distributed.

To characterize this criticality behavior, Ding et al. used a generalized forest fire model. In this model, new trees grow in a forest with a probability $p$, while lightning strikes-which start fires that burn down closely packed trees-occur at a rate $r$. This simple model-balancing tree growth (drive) and tree destruction (dissipation) - can explain why tree clusters in a forest have a range of sizes that follow a power law. In the Rydberg analogy, the laser-excitation of an atom represents the growth of a new tree. Occasionally, a cluster of atoms reaches a high-density threshold, corresponding to a lightning strike, at which point that cluster and all the nearby clusters "catch fire" and burn down to the ground state. The team showed that, for specific values of $p$ and $r$, this self-organizing model reproduced the observed Rydberg atom densities in the experiment.

These experimental results offer a fresh new perspective on a venerable problem and open up a broad range of questions that can be addressed in a controlled way using the enormous versatility of Rydberg gases. Long standing problems in the field, such as the universal and nonuniversal aspects of self-organized criticality and its relations to nonequilibrium phase transitions, could be experimentally addressed. Given the broad impact that this principle has had beyond physics, this platform has the potential to become a controllable environment to study analog dynamical systems relevant to a broad range of disciplines.

This research is published in Physical Review X.

Correction (29 April 2020) : The article was updated to indicate the contribution from Dong-Sheng Ding and colleagues at the University of Science and Technology of China in Hefei.

\section{REFERENCES}

[1] D.-S. Ding et al., "Phase diagram and self-organizing dynamics in a thermal ensemble of strongly interacting Rydberg atoms," Phys. Rev. X 10, 021023 (2020).

[2] K. G. Wilson, "Problems in physics with many scales of length," Sci. Am. 241, 158 (1979).

[3] P. Bak et al., "Self-organized criticality: An explanation of the $1 / f$ noise," Phys. Rev. Lett. 59, 381 (1987).

[4] B. Drossel and F. Schwabl, "Self-organized critical forest-fire model," Phys. Rev. Lett. 69, 1629 (1992).

[5] N. W. Watkins et al., "25 Years of self-organized criticality: Concepts and controversies," Space. Sci. Rev. 198, 3 (2015); M. A. Muñoz, "Colloquium: Criticality and dynamical scaling in living systems," Rev. Mod. Phys. 90, 031001 (2018).

[6] A. Vespignani and S. Zapperi, "Order parameter and scaling fields in self-organized criticality," Phys. Rev. Lett. 78, 4793 (1997); J. A. Bonachela and M. A. Muñoz, "Self-organization without conservation: True or just apparent scale-invariance?" J. Stat. Mech. 2009, P09009 (2009); R. Dickman et al., "Paths to self-organized criticality," Braz. J. Phys. 30, 27 (2000).

[7] M. Saffman et al., "Quantum information with Rydberg atoms," Rev. Mod. Phys. 82, 2313 (2010).

[8] H. Weimer et al., "A Rydberg quantum simulator," Nat. Phys. 6, 382 (2010); T. L. Nguyen, "Towards quantum simulation with circular Rydberg atoms," Phys. Rev. X 8, 011032 (2018); A. Browaeys and T. Lahaye, "Many-body physics with individually controlled Rydberg atoms," Nat. Phys. 16, 132 (2020).

[9] S. Helmrich et al., "Signatures of self-organized criticality in an ultracold atomic gas," Nature 577, 481 (2020).

10.1103/Physics. 13.70 